\title{
Rheumatoid Factors Isolated from Patients with Autoimmune Disorders Are Derived from Germline Genes Distinct from Those Encoding the Wa, Po, and Bla Cross-Reactive Idiotypes
}

\author{
Kimberly D. Victor, ${ }^{*}$ Ingrid Randen, ${ }^{* 5}$ Keith Thompson, Oystein Forre, Jacob B. Natvig, ${ }^{\ddagger}$ Shu Man Fu," and J. Donald Capra \\ ${ }^{*}$ Department of Microbiology, University of Texas Southwestern Medical Center, Dallas, Texas 75235-9048; ${ }^{\ddagger}$ University of Oslo, \\ Institute of Immunology and Rheumatology and the Rheumatism Hospital, Oslo 1, Norway; ${ }^{\S}$ RC, MIP Unit and Department of \\ Immunology, Institute of Animal Physiology and Genetics Research, Cambridge CB2 4AT, United Kingdom; and "Division of \\ Rheumatology, Department of Medicine, University of Virginia School of Medicine, Charlottesville, Virginia 22908
}

\begin{abstract}
To better understand the structural basis for rheumatoid factor activity, the nucleotide sequence of the light chain variable regions of nine human monospecific IgM rheumatoid factors were analyzed. Rheumatoid factors were isolated from three patients with rheumatoid arthritis, a patient with systemic lupus erythematosus, and a normal individual. The $V_{L}$ gene segments used by these rheumatoid factors are not as restricted as previous work on mixed cryoglobulin rheumatoid factors had suggested. Each of the different $V_{K}$ families is represented and there are two examples where $a V_{\lambda}$ gene segment is used. Molecules with structures similar to those of the Wa and Po CRI, characteristic of mixed cryoglobulin rheumatoid factors, are not common among these rheumatoid factors isolated from patients with rheumatoid arthritis. While there are clear examples of rheumatoid factors that are direct copies of germline genes, most of the sequence data suggest that the processes of antigenic selection and somatic mutation contribute significantly to the generation of monospecific rheumatoid factors in patients with autoimmune disease. (J. Clin. Invest. 1991. 87:1603-1613.) Key words: human autoantibody • rheumatoid factors
\end{abstract}

\section{Introduction}

Rheumatoid factors are autoantibodies that react with the $F_{c}$ region of $\operatorname{IgG}(1-3)$. The synthesis of rheumatoid factors is well documented during many secondary immune responses and under these circumstances, rheumatoid factors are thought to play a physiological role in the clearance of circulating immune complexes. Rheumatoid factor production is also characteristic of several autoimmune disorders such as rheumatoid arthritis, Sjogren's syndrome, mixed connective tissue disease, and systemic lupus erythematosus. It is not known whether the antibodies produced in these autoimmune diseases play a pathogenic role. However, in rheumatoid arthritis, higher titers of rheumatoid factors in the serum are known to correlate with

Address correspondence to Dr. J. Donald Capra, Department of Microbiology, University of Texas Southwestern Medical Center, 5323 Harry Hines Boulevard, Dallas, TX 75235-9048.

Received for publication 17 May 1990 and in revised form 7 October 1990.

J. Clin. Invest.

(c) The American Society for Clinical Investigation, Inc.

$0021-9738 / 91 / 05 / 1603 / 11 \$ 2.00$

Volume 87, May 1991, 1603-1613 a more aggressive form of the disease $(4,5)$. There is also evidence for rheumatoid factor production in the affected joints.

Most of the available information regarding the primary structure of human rheumatoid factors comes from studies of IgM paraproteins with rheumatoid factor activity isolated from patients with mixed cryoglobulinemia (6). Early work showed that the light chains of these rheumatoid factors were structurally similar and probably derived from a limited number of $V_{K}$ gene segments (7). A large body of experimental evidence documents that the majority of these rheumatoid factors belong to three cross-reactive idiotypic (CRI) ${ }^{1}$ groups, Wa, Po, and Bla (8-17). The major CRI present on $\sim 65 \%$ of mixed cryoglobulin rheumatoid factors is the Wa idiotype. In addition to polyclonal sera, Wa idiotope positive molecules usually react with MAb 17.109 that recognizes determinants encoded by a specific $V_{K}$ IIIb germline gene segment termed HumKv325 (16-18). It has also been shown that Wa idiotypepositive rheumatoid factors are associated preferentially $\left(>75.0 \%\right.$ ) with heavy chains expressing a specific $\mathrm{V}_{\mathrm{H}} \mathrm{I}$ gene segment recognized by the MAb G6 (16). The Po CRI is present on $\sim 15 \%$ of mixed cryoglobulin rheumatoid factors. This idiotypic marker is generally encoded by a $V_{K}$ IIIa gene segment (HumKv328) recognized by the MAb 6B6.6 (19). In rheumatoid factors this particular light chain is typically associated with $\mathrm{V}_{\mathbf{H}}$ III variable region genes. The Bla CRI is expressed on $\sim 15 \%$ of mixed cryoglobulin rheumatoid factors and detects an idiotope encoded by the same $V_{K}$ IIIa gene segment (HumKv328) as the Po CRI but Bla positive molecules are generally associated with heavy chains expressing $\mathrm{V}_{\mathrm{H}} \mathrm{IV}$ variable region genes (20).

As rheumatoid factors isolated from mixed cryoglobulins generally bear one of these three major idiotypes, it was originally thought that conserved genes encode many if not all rheumatoid factor light chains regardless of origin. However, while the Wa and Po CRI have been detected in polyclonal rheumatoid factors present in the sera of patients with rheumatoid arthritis (21-24), the amounts of these CRI are quite modest. Extensive serologic analyses of polyclonal rheumatoid factors from patients with rheumatoid arthritis show them to be diverse, and that the common idiotypes of the mixed cryoglobulin rheumatoid factors account for only a minor population $(25,26)$.

This study characterizes the molecular structures of the light chains of nine monoclonal, monospecific rheumatoid factors derived from the inflamed synovial tissue of two patients with rheumatoid arthritis, a patient with polyarticular juvenile

1. Abbreviations used in this paper: $\mathrm{CDR}$, complementary determining region; CRI, cross-reactive idiotypic; FW, framework; PCR, polymerase chain reaction. 
arthritis, a patient with systemic lupus erythematosus, and a normal individual. We reasoned that the use of synovial tissue from the site of active inflammation as a source of B-lymphocytes is more likely to produce rheumatoid factors that accurately reflect the genetic origin of antibodies produced in rheumatoid arthritis.

This study documents that the $\mathrm{V}_{\mathrm{L}}$ gene segment repertoire used by monoclonal rheumatoid factors in disease states is neither restricted to particular $V_{L}$ germline gene segments nor is it related to the repertoire believed to encode the majority of rheumatoid factors isolated from patients with mixed cryoglobulinemia.

\section{Methods}

Generation and serological characterization of human monoclonal IgM rheumatoid factors. The two monospecific rheumatoid factors RFKES1 and RF-TMC1 were isolated from EBV-transformed peripheral blood B-lymphocytes obtained from a patient with systemic lupus erythematosus (KES) and a normal individual (TMC). These cell lines were derived by EBV transformation of a limited number of non-T lymphocytes as described (27) and subsequently single cell cloned by limiting dilution. The remaining seven rheumatoid factors were isolated from EBV-transformed B-lymphocytes obtained from the synovial tissue of two patients with rheumatoid arthritis (TS and SJ) and one patient with polyarticular juvenile rheumatoid arthritis $(\mathrm{KL})(28)$. Each cell line was fused with the murine myeloma (X63-Ag8.653) and cloned by limiting dilution. Clones RF-TS1, RF-TS2, and RF-TS3 derive from patient TS. RF-SJ1, RF-SJ2, and RF-SJ3 were obtained from patient SJ, and RF-KLl was isolated from patient $\mathrm{KL}$.

RF-KES1 and RF-TMC1 are monospecific rheumatoid factors that are reactive with human IgG but not rabbit IgG by ELISA. RF-KESI reacts predominantly with $\operatorname{IgGl}$ and IgG3 with some reactivity with IgG2. RF-TMC1 reacts strongly with IgG1 and weakly with IgG3 and IgG4 (Fu, S. M., unpublished data). The specificities for the remaining rheumatoid factors have been described elsewhere $(28,29)$. Briefly, each of these hybridomas represent monospecific rheumatoid factors that recognize human IgG. In addition, RF-SJ1 and RF-TS2 also react with rabbit IgG. By using various IgG subclass myeloma proteins of different $\mathrm{Gm}$ allotypes, the fine specificities of these antibodies were determined. The antibodies RF-SJ1 and RF-TS1 represent pan-specific rheumatoid factors. The antibodies RF-TS2, RF-TS3, RF-SJ2, and RF-SJ3 exhibit the classical Ga reactivity pattern and the rheumatoid factor RF-KL1 is an example of an antibody that exhibits the new $\mathrm{Ga}$ related specificity.

Amplification and isolation of the $V_{L}$ gene segments. To isolate the $\mathrm{V}_{\mathrm{K}}$ gene segments, 2-20 $\mu \mathrm{g}$ of total RNA was reverse transcribed using avian myeloblastosis viral reverse transcriptase into first strand cDNA using oligo $d(T)$ as the primer in a $50-\mu l$ reaction volume (30). Second strand synthesis and amplification were carried out using the polymerase chain reaction (PCR) on 1-3 $\mu$ l of the first strand cDNA reaction material in a final vol of $100 \mu$ l containing $10 \mathrm{mM}$ Tris- $\mathrm{HCl}, \mathrm{pH} 8.8,1$ $\mathrm{mM} \mathrm{MgCl} 2,2 \mathrm{mM}\left(\mathrm{NH}_{4}\right)_{2} \mathrm{SO}_{4}, 1 \mathrm{mM}$ 2-mercaptoethanol, $0.5 \mathrm{mM}$ EDTA, pH 8.0, $200 \mu \mathrm{M}$ of each dNTP, $2.5 \mathrm{U}$ Taq polymerase, and 50 $\mu \mathrm{M}$ of each primer $(31,32)$. The oligonucleotides specific for the $5^{\prime}$ portion of the $V_{K} I, V_{K} I I$, and $V_{K} I I I$ families consisted of 20 -mer encoding amino acid residues -16 to -10 . The leader sequence specific primers are $5^{\prime}$ CTCAGCTCCTGGGGCTCCTG $3^{\prime}$ for the $V_{k} I$ and $\mathrm{V}_{\mathbf{K}} \mathrm{II}$ families and 5' CTCCTGCTACTCTGGCTC $3^{\prime}$ for the $\mathrm{V}_{\mathrm{K}} \mathrm{III}$ family. The oligonucleotide specific for the $3^{\prime}$ end consisted of a 23 mer from the kappa constant region gene with the sequence $5^{\prime}$ TCTAGATCATCAGATGGCGGGAA 3'.

For the $V_{\lambda}$ gene segments, the same general procedure was followed to generate first strand cDNA. Second strand synthesis was accomplished following the Okayama and Berg procedure (33). The double stranded cDNA was blunt ended using the Klenow fragment and ligated into EcoRV digested, dephosphorylated plasmid (pBluescript $\mathrm{KS}+$ ) in $30 \mu \mathrm{l}$ total reaction vol. Amplification was carried out using
1-3 $\mu$ l of the ligation mixture using the PCR under conditions described above. The oligonucleotide specific primer for the $5^{\prime}$ end made use of the T7 promoter sequences present within the vector and consisted of a 20 mer with the sequence 5' TAATACGACTCACTATAGGG $3^{\prime}$. A portion of the lambda constant region gene $\left(C_{\lambda} I\right)$ was used as the $3^{\prime}$ specific oligonucleotide primer with the sequence $5^{\prime}$ CTCGAGTGTGGCCTTGTTGGCTTG 3 '.

40 cycles of amplification were performed using the following conditions: denaturation for $1 \mathrm{~min}$ at $94^{\circ} \mathrm{C}$, annealing for $2 \mathrm{~min}$ at $56^{\circ} \mathrm{C}$, and extension for $1 \mathrm{~min}$ at $72^{\circ} \mathrm{C}$. PCR products of the appropriate size were purified by electroelution. The isolated fragment was phosphorylated using T4 polynucleotide kinase and ligated into EcoRV digested, dephosphorylated vector (pBluescript $\mathrm{KS}+$ ). The ligation mixture was used to transform BSJ-72 competent cells and the resulting colonies were screened with oligonucleotides corresponding to a conserved region of framework 3 which were end labelled with ${ }^{32} \mathrm{P}$-gamma ATP and T4 polynucleotide kinase. Single strand DNA was prepared from positive colonies and sequenced using dideoxy chain termination sequencing procedures with ${ }^{35} \mathrm{~S}$-alpha-thio ATP and Sequenase $(34,35)$. Several independent clones were sequenced to verify the results.

Amplification and analysis of genomic DNA by specific oligonucleotide probes. Genomic DNA from different patients and normal individuals was prepared from peripheral blood lymphocytes as well as from normal liver. Using oligonucleotide primers corresponding to amino acid positions $1-6$, specific for the $5^{\prime}$ portion of each variable region gene studied, and a primer specific for amino acid residues $62-68$ as a 3 ' specific primer for the PCR, given variable region gene subsets were amplified using the PCR conditions stated above in $100 \mu$ l total vol. A $10-\mu 1$ aliquot of each PCR reaction was electrophoresed on an $0.8 \%$ agarose gel and transferred onto Zetaprobe (36). The filters were hybridized with specific complementary determining region (CDR) or framework (FW) specific oligonucleotides which had been end labelled with ${ }^{32} \mathrm{P}$-gamma ATP and T4 polynucleotide kinase. Filters were washed in $6 \times \mathrm{SSC}, 0.1 \% \mathrm{SDS}$ at $2^{\circ} \mathrm{C}$ below the $\mathrm{T}_{\mathrm{m}}$ (melting temperature) for each screening oligonucleotide for $30 \mathrm{~min}$ and exposed for $\sim 30 \mathrm{~min}$ on Kodak XAR film.

In the analysis of each rheumatoid factor several controls were included. As a positive control, DNA from each respective cell line was amplified using the same conditions as were used for the DNA prepared from the peripheral blood and the liver. As a negative control, DNA from antibodies that use the same $V_{L}$ gene family but differ at the regions used for screening oligomers were tested to ensure that the hybridization conditions would detect at least a one or two nucleotide difference. After hybridization, if a negative result was detected with the peripheral blood and liver DNA, the blots were stripped and reprobed with an internal oligomer that detects a conserved region within FW3 to rule out the possibility that the variable region subset was not amplified in the PCR reaction thus generating a negative result.

The screening oligomers used for each antibody are as follows: (a) RF-KES1 studies used a CDR 1 probe corresponding to amino acids 27-34 and a FW3 probe corresponding to amino acids 74-79. (b) RFKL1 studies used a CDR 1 probe corresponding to amino acids 27-34 and a CDR3 probe corresponding to amino acids $90-95$. In addition, an FW2 oligomer from region 43-49 was tested. (c) RF-TS1 studies used a CDR3 probe generated from amino acids $92-97$ and an FW3 probe corresponding to amino acids 84-89. (d) RF-TS2 studies used probes from CDR1 (amino acids 27-33), CDR3 (amino acids 91-96), and an FW3 probe from region 84-89. (e) RF-TMCl studies used a CDR3 probe corresponding to amino acids $90-97$, a FW 1 probe corresponding to amino acids $13-18$, and a FW3 probe corresponding to amino acid residues $72-77$. $(f)$ RF-SJ1 studies used a CDR 1 probe generated from amino acids 25-33. (g) RF-SJ2 studies used a CDR1 probe also from the region containing amino acids $25-33$. In each experiment, where several screening oligomers were used to examine a particular $V_{L}$ subset of a given antibody, the results were consistent for all the probes used. In this regard, the use of FW probes as screening oligomers may be misleading as framework regions are generally conserved. However, the use of CDR region screening oligomers confirmed in each case the results obtained with the FW probes. 
The analysis of the $\mathrm{J}_{\mathrm{K}} \mathrm{V}$ locus of patient TS was conducted in a similar manner. The genomic $J_{K} V$ gene segment was amplified using oligomers corresponding to the $5^{\prime}$ and $3^{\prime}$ flanking regions of this locus. To ensure that the product was representative of the genome, a screening oligomer corresponding to the intron between the heptamer and nonamer recombination sequences was used in addition to the screening oligomers corresponding to the published genomic sequence of $J_{K} V$ and the "allelic" variant present in this patient.

\section{Results}

Two rheumatoid factors use $V_{K} I$ gene segments that are distinct from the $V_{K} I$ gene segments used in mixed cryoglobulin rheumatoid factors. Four different $\mathrm{V}_{\mathrm{L}}$ region subfamilies were used by the nine monoclonal rheumatoid factors (Table I). The $V_{L}$ gene segments of two monospecific rheumatoid factors belong to the $V_{K} I$ family. RF-KES1 was isolated from a patient with systemic lupus erythematosus and uses $V_{K} I$ and $J_{K} V$ light chain gene segments in association with a $V_{H}$ III heavy chain gene segment (Fig. 1 and Table I). Although this particular heavy and light chain combination has been previously characterized in some Po-positive rheumatoid factors, the $V_{L}$ region gene of RF-KES1 is most closely related to the light chain of the GAL rheumatoid factor with $88.7 \%$ homology at the amino acid level (data not shown). RF-KL1, a rheumatoid factor isolated from a patient with juvenile rheumatoid arthritis, also expresses $V_{K} I$ and $J_{K} V$ light chain gene segments in association with a $\mathrm{V}_{\mathrm{H}}$ III heavy chain gene (Fig. 1 and Table I), again characteristic of some Po-positive rheumatoid factors, and shares 92.7\% homology to the $V_{L}$ region of the GAL rheumatoid factor (data not shown). At the nucleotide level, RF-KES1 is $96.0 \%$ identical to the germline gene V52 whereas RF-KL1 is 97.5\% identical to the germline gene Vd (Fig. 2 and Table I). Although RF-KESI and RF-KLl are derived from different germline genes and share a limited degree of homology, both RF-KES1 and RF-KL1 are very similar within CDR1 and CDR2 (one amino acid substitution in CDR1) suggesting that these regions may play some role in rheumatoid factor activity.

To determine if these rheumatoid factors were germline encoded, Southern analysis was performed using amplified variable region gene subsets from the respective antibodies as positive controls, as well as from peripheral blood lymphocytes from a number of normal individuals that were hybridized with oligonucleotide probes specific for unique sequences within the CDR or FW regions. Thus, RF-KES1 represents a rheumatoid factor that is either encoded by a germline gene unique to the patient KES or an antibody that has undergone somatic mutation, as CDR1 and FW3-specific probes failed to detect a hybridizing variable region gene fragment in a number $(n=20)$ of normal individuals. RF-KL1, however, is probably germline encoded as genomic DNA from each of the normal individuals tested $(n=20)$ produced a variable region gene fragment capable of hybridizing to CDR1, CDR3, and FW2-specific probes. Thus, these results suggest that one rheumatoid factor, RF-KES1, has undergone somatic mutation, while the rheumatoid factor RF-KLl is probably germline encoded with only a limited amount of somatic variation.

Characterization of a $V_{K} I I$ rheumatoid factor identical at the nucleotide level to the $V_{K} I I$ germline gene A23. RF-TS3 expresses a $V_{K} I I$ gene segment and represents the first such rheumatoid factor reported. This antibody was isolated from a patient with rheumatoid arthritis and uses $V_{K} I I$ and $J_{K} V$ gene segments in association with a $V_{H} I$ variable region gene (Fig. 3 and Table I). The variable region gene segment is identical at the nucleotide level to the $V_{K}$ II germline gene A23 (Fig. 2 and Table I) and represents a clear example of a germline gene that directly encodes an autoreactive antibody. It is interesting that the entire sequence of RF-TS3 is germline encoded except for the last two amino acid residues within CDR3 that are contributed by the $\mathrm{J}$ segment. As it is unlikely that somatic mutation or $\mathrm{N}$-segment addition could account for the four nucleotide changes that result in the amino acid substitutions at positions 101-102 of arginine and asparagine residues for isoleucine and threonine residues, respectively, (which are encoded within the germline $J_{K} V$ gene segment), this suggested that patient TS possessed an allelic variant at the $\mathrm{J}_{\mathbf{K}} \mathrm{V}$ locus. Using oligonucleotides specific for these amino acid differences as well as for the germline sequence, genomic $J_{K} V$ segments from patient TS and from a number of normal individuals $(n=4)$ were analyzed. Patient TS was found to have $J_{K} V$ segments that corresponded to both the germline and allelic forms of this locus (data not shown). The allelic variant of this locus was detected in several other individuals and may represent a common polymorphism not previously noted. Analysis of RF-TS3 clearly demonstrates that the expression of certain germline variable region gene segments in unmutated form may be isolated from patients with autoimmune diseases such as rheumatoid arthritis.

Table I. Homology between Rheumatoid Factors and Germline/Expressed Light Chain Gene Segments

\begin{tabular}{|c|c|c|c|c|c|}
\hline \multirow[b]{2}{*}{ Antibody } & \multirow[b]{2}{*}{ Class } & \multirow{2}{*}{$\begin{array}{c}\text { Related } V_{L} \\
\text { gene segment }\end{array}$} & \multicolumn{2}{|c|}{ Percent identity } & \multirow{2}{*}{$\begin{array}{l}V_{H} \text { Gene } \\
\text { segment* }\end{array}$} \\
\hline & & & Nucleotide level & Amino acid level & \\
\hline RF-KES1 & IgM, $V_{K} I$ & V52 & 96.0 & 94.6 & $\mathrm{~V}_{\mathrm{H}}$ III \\
\hline RF-KL1 & IgM, $V_{K} I$ & Vd & 97.5 & 96.8 & $\mathrm{~V}_{\mathrm{H}} \mathrm{III}$ \\
\hline RF-TS3 & IgM, $V_{\mathbf{K}}$ II & A23 & 100.0 & 100.0 & $\mathbf{V}_{\mathbf{H}} \mathbf{I}$ \\
\hline RF-TS1 & IgM, $\mathrm{V}_{\mathbf{K}}$ IIIb & Humkv325 & 99.3 & 100.0 & $\mathbf{V}_{H} \mathbf{I}$ \\
\hline RF-TS2 & IgM, $V_{\mathbf{K}}$ IIIa & Humkv328 & 97.0 & 97.0 & $\mathrm{~V}_{\mathrm{H}} \mathrm{III}$ \\
\hline RF-TMCl & IgM, $V_{\mathbf{K}}$ III & $38 \mathrm{~K}$ & 93.8 & 88.5 & $\mathrm{~V}_{\mathrm{H}} \mathrm{IV}$ \\
\hline RF-SJ3 & IgM, $\mathrm{V}_{\mathrm{I}} \mathrm{IIIb}$ & Humkv325 & 98.7 & 98.0 & $\mathrm{~V}_{\mathbf{H}}$ III \\
\hline RF-SJ1 & $\operatorname{IgM}, V_{\lambda} I$ & FOG-B** & 93.0 & 88.0 & $\mathrm{~V}_{\mathrm{H}} \mathrm{III}$ \\
\hline RF-SJ2 & $\operatorname{IgM}, \mathbf{V}_{\lambda} \mathbf{I}$ & FOG-B** & 96.3 & 92.0 & $\mathrm{~V}_{\mathrm{H}} \mathrm{III}$ \\
\hline
\end{tabular}

* Pascual, V., personal communication, and reference $41 .{ }^{* *}$ FOG-B is an expressed $V_{\lambda} I$ anti-D (Rh) antibody. 
RF-KES1 GACATCCAGATGACCCAGTCTCCATCCTCCCTGTCTGCATCTGTAGGAGACAGAGTCACCATCACTTGCCGgGCGATCAGGGCATTAGGAACTATTIAG

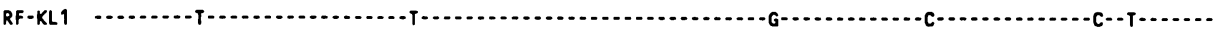

CDR2

RF-KES1 CCTGGTATCAGCAGAACCAGGGAAGTCCCTAAACTCCTGATCTATGCTGCATCCACTTTGCAATCAGGGGTCCCATCTCGGTTCAGCGGCAGTGCATC

RF-KL1

CDR3

RF-KES1 TGGGACAGAGTTCACTCTCACCATCAGCAGCCTGCAACCTGAAGATGTTGCAACTTATTACTGTCAAAAGTATAACACTGCCCCCCCTCAC

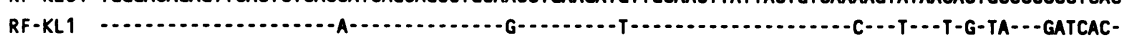

B

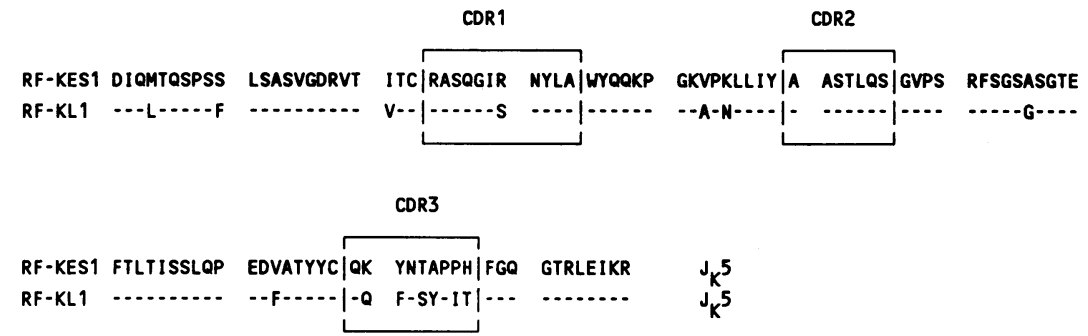

Figure 1. (A) Nucleotide sequence comparison of the $V_{L}$ gene segments of the $V_{K} I$ rheumatoid factors RF-KES1 and RF-KL1. Dashes represent identical nucleotide sequences. The CDR regions are indicated. $(B)$ Comparison of the translated amino acid sequences of the RFKES1 and RF-KL1 rheumatoid factors. Dashes indicate identical amino acid sequences. Each of the CDR regions are presented within the outlined areas, and the $J$ segment used is shown.
Four monospecific monoclonal rheumatoid factors use different $V_{K} I I I$ gene segments. RF-TS1 is a classical Wa-positive rheumatoid factor. It expresses the antigenic determinant defined by the CRI MAb 17.109 and uses an MAb G6-positive $\mathrm{V}_{\mathrm{H}} \mathrm{I}$ heavy chain gene $(28,29$, and Table $\mathrm{I})$. This antibody expresses $V_{K} I I I b$ and $J_{K} I$ gene segments (Fig. 4) and is identical at the amino acid level to the light chain of the BOR rheumatoid factor (data not shown). As this antibody is an example of a Wa-positive rheumatoid factor, it is not surprising that it shares the greatest homology, $\sim 99.3 \%$, at the nucleotide level and is identical at the amino acid level to the germline gene HumKv325, which is believed to encode this particular CRI (Fig. 2 and Table I). This suggests that the variable region gene used in RF-TS1 is either encoded by the HumKv325 germline gene or a closely related gene. Southern analysis using CDR3 and FW3 screening oligomers supported this conclusion.

RF-TS2 was isolated from a patient with rheumatoid arthritis and uses $V_{K}$ III and $J_{K} V$ gene segments in association with a $\mathrm{V}_{\mathrm{H}}$ III heavy chain gene (Fig. 4 and Table I). RF-TS2 shares the most homology with the $V_{L}$ region gene segment of the POM rheumatoid factor $(89.7 \%)$. When RF-TS2 is compared to POM, these two rheumatoid factors are identical within CDR3, suggesting that this region of the antibody may be important in rheumatoid factor activity (data not shown). The POM rheumatoid factor expresses the Po CRI and uses a $\mathrm{V}_{\mathrm{K}}$ IIIa gene segment recognized by the MAb 6B6.6 and is believed to be encoded by the germline HumKv328 gene segment. While RF-TS2 is closely related to this rheumatoid factor, it does not react with MAb 6B6.6, suggesting that the epitope seen by this CRI antibody lies outside CDR3. At the nucleotide level, RF-TS2 shares the most homology $(97.0 \%)$ with the germline variable region gene HumKv328. Similar to previous studies with RF-KES1 and RF-KL1, Southern analysis of amplified subsets of variable region gene segments was performed using CDR 1, CDR3, and FW3 specific oligonucleotide probes. These probes were able to detect from peripheral blood lymphocytes from the donor and several normal individ- uals $(n=20)$ a specific variable region gene fragment suggesting that RF-TS2 is germline encoded, the most likely candidate being HumKv328 or a closely related gene (Fig. 2 and Table I).

The most interesting feature of this antibody is that within CDR3 at amino acid position 96, there is the addition of an extra proline residue. While the length of CDR1 and CDR2 to a degree can vary in kappa light chain genes, CDR3 is classically nine amino acid residues in length. The first seven amino acids are contributed by the particular variable region gene segment and the last two amino acids are encoded by the joining region gene segment. RF-TS 2 is therefore one of a limited number of examples where this variation has been characterized.

Rheumatoid factor RF-TMC1 represents an antibody isolated from a normal individual that uses $V_{K} I I I$ and $J_{K} I V$ gene segments in combination with a $V_{H} I V$ heavy chain gene (Fig. 4 and Table I). At the expressed level of the light chain, RFTMC1 is most closely related to the rheumatoid factor RIV, that bears the Wa CRI, and shares $88.7 \%$ homology at the amino acid level (data not shown). The variable region gene used in this antibody is structurally distinct and cannot be categorized as belonging to either the $\mathrm{V}_{K}$ IIIa or the $\mathrm{V}_{\mathbf{K}}$ IIIb subfamilies. The sequence encoding amino acid residues 13-18 in FW1 as well as amino acid residues 72-77 in FW3 are unusual and have not been described previously. Also, CDR3 contains a number of amino acids that are not typically expressed in mixed cryoglobulin rheumatoid factors characterized up until this point. As variations in FW regions within a given subfamily are infrequent in the absence of somatic mutation, the sequence differences seen in FW1, FW3, and CDR3 suggest that RF-TMC1 has undergone somatic diversification. At the genomic level, RF-TMC1 is most closely related to the germline variable region gene $38 \mathrm{~K}$ (Fig. 2 and Table I), isolated by Hillson et al. (personal communication), a variable region gene expressed in a fetal cDNA library. These two antibodies share 93.8\% homology at the nucleotide level and share several structural similarities. The similarities within CDR3 of these two 

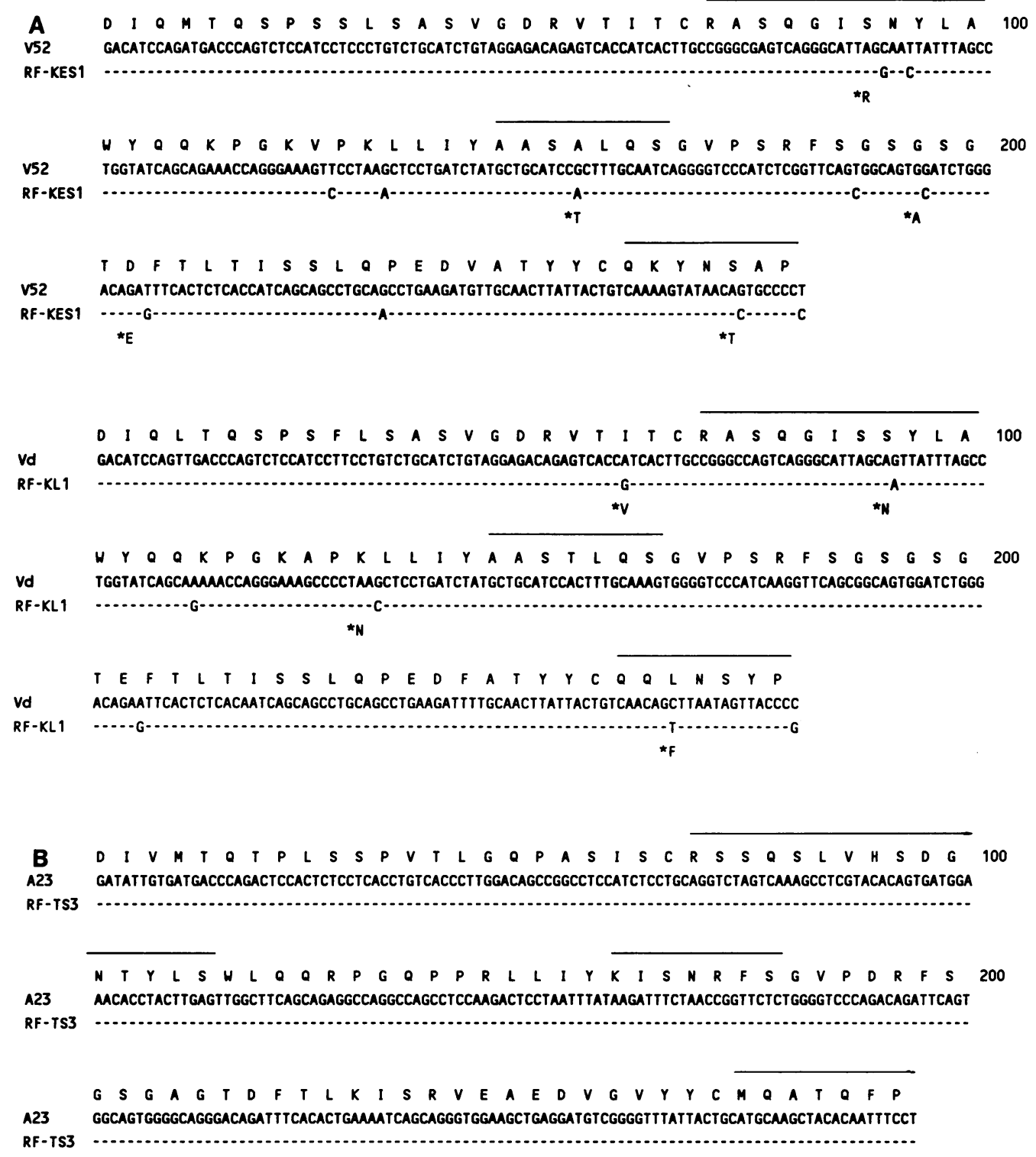

Figure 2. Comparison of the nucleotide sequences of the $V_{L}$ gene segments of each of the rheumatoid factors with their most homologous germline gene. In the comparison shown for the $V_{\lambda} I$ rheumatoid factors RF-SJ1 and RF-SJ2, the antibody FOG-B represents the most homologous expressed $V_{\lambda} I$ gene segment as there are few human $V_{\lambda}$ germline gene sequences available. The translated amino acid sequence of each germline or expressed gene is presented at the top of each comparison, and amino acid differences present in the respective rheumatoid factor are shown below the figure and are indicated by an asterisk $\left({ }^{*}\right)$. Dashes indicate areas of identity and the CDR regions are G S G T D F T L T I S R L E P E Humkv325 GGGTCTGGGACAGACTTCACTCTCACCATCAGCAGACTGGAGCCTGAaGATTTTGCAGTGTATTACTGTCAGCAGTATGGTAGCTCACCT RF-TS1 enclosed.

antibodies are striking. As mentioned previously, CDR3 segments tend to have no variation in length, yet both $38 \mathrm{~K}$ and RF-TMC1 contain CDR3 segments of 10/11 amino acids, respectively. $38 \mathrm{~K}$ expresses an additional proline residue at position 96 and RF-TMC1 contains this same proline residue at position 96 and a second proline residue at position 97 . The similarity of these two variable region genes suggests a common origin from $38 \mathrm{~K}$ or a closely related gene.

To determine if RF-TMC1 uses a germline-encoded variable region gene, FW1, FW3, and CDR3-specific oligonucleotide probes were designed and used in Southern analysis to screen peripheral blood lymphocytes from a large number of 
C E I V M T O S P A T L S V S P G E R A T L S C R A S Q S V S S N 100

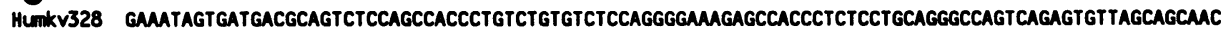
RF-TS2

L A H Y Q Q K P G Q A P R L L I Y G A S T R A T G I P A R F S G S 200 Humkv328 TTAGCCTGGTACCAGCAGAACCTGGCCAGGCTCCCAGGCTCCTCATCTATGGTGCATCCACCAGGGCCACAGGCATCCCAGCCAGGTTCAGTGGCAGT RF-TS2

G S G T E F T L T I S S L Q S E D F A V Y Y C Q Q Y Y N N

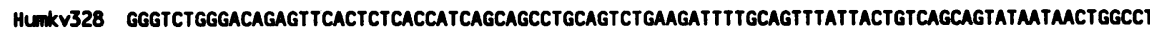
RF-TS2
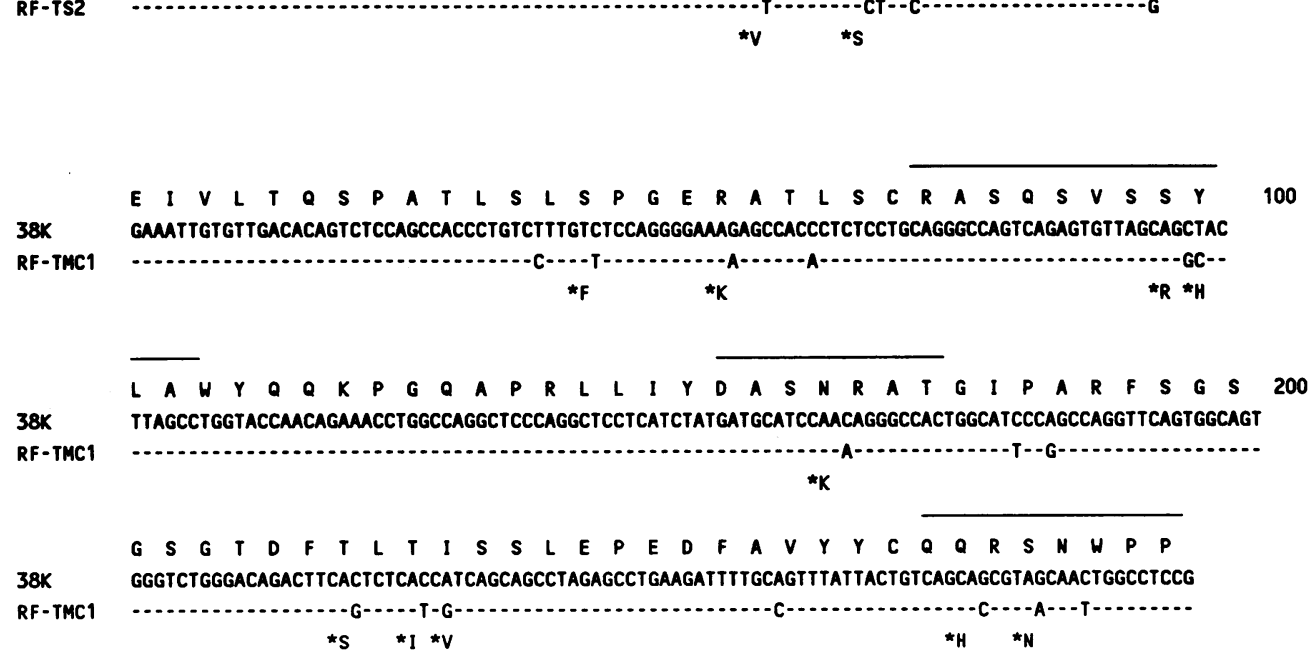

D E I V L T Q S P G T L S L S P G E R A T L S C R A S Q S S $V$ S $S$ S $S$ S $Y$ S 100

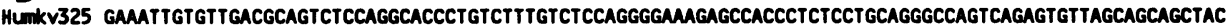
RF-SJ3

L A W Y Q Q K P G Q A P R L L I Y G A S S R A T G I P D R F S G S 200 Hunkv325 tTAGCCTGGTACCAGCAGMACCTGGCCAGGCTCCCAGGCTCCTCATCTATGgTGCATCCAGCAGGGCCACTGGCATCCCAGACAGGTTCAGTGGCAGT RF-SJ3 $\star R$

G S G T D F T L T I S R L E P E D F A V Y Y

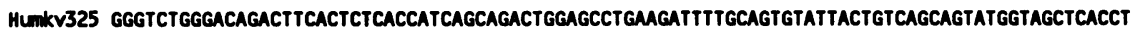
RF-SJ3

Q S V L T Q P P S V S A A P G Q K V T I S C S G T S S N I G N N Y V S 100 FOG-B CAGTCTGTGTTGACGCAGCCGCCCTCAGTGTCTGCGGCCCCAGGACAGAGGTCACCATCTCCTGCTCCGGACCAGCTCCMCATTGGGMTMUTAATGTATCC RE-

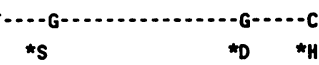

W Y Q Q L L FOG-B TGGTATCAGCAGCTCCCAGgMCAGCCCCCMACTCCTCATTTATGACAATAMTAMGCGACCCTCAGGGATTCCTGACCGATTCTCTGGCTCCMGGTCTGGCACGTCA RF-SJ2

A T L G I T G L R $T$ G D E A $D$ S Y Y C C A T FOG-B GCCACCCTGGGCATCACCGGACTCCGGACTGGGGACGAGGCCGATTATTACTGCGCAACATGGGATAGCAGCCTGAGTGCTGTGGTG RF-SJ2 $-\cdots, \ldots$ $\star \mathbf{G}$

Figure 2 (Continued)

normal individuals $(n=50)$. None of these probes detected an appropriate variable region fragment in normal individuals suggesting that somatic diverification has occurred in this antibody.
A second rheumatoid factor, RF-SJ3 isolated from a patient with rheumatoid arthritis, was also found to express the Wa CRI defined by the MAb 17.109. While this antibody uses $V_{K}$ IIIb and $J_{K}$ II gene segments (Fig. 4), the heavy chain gene 


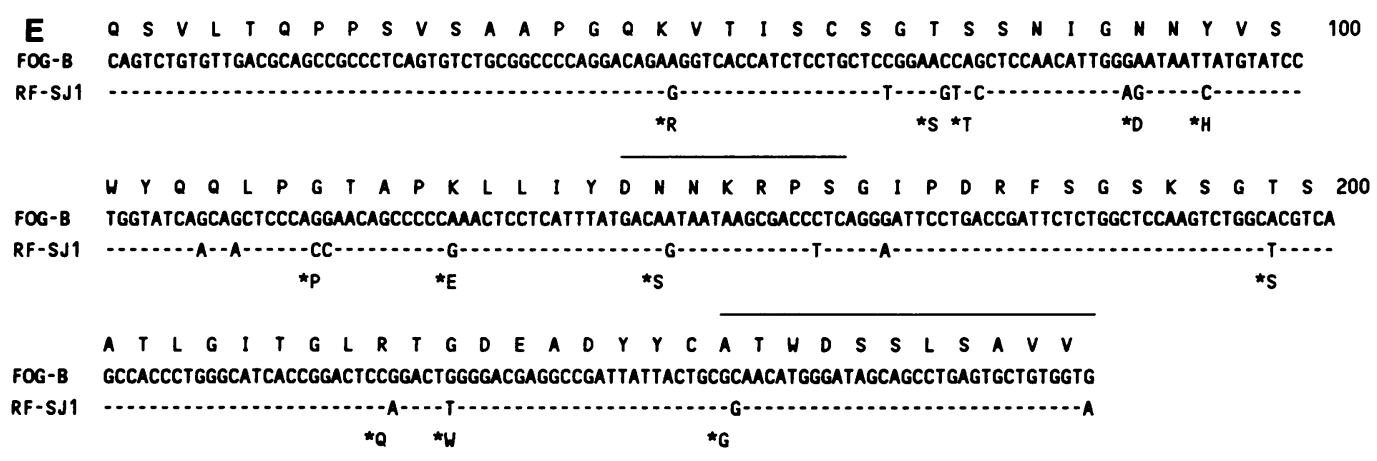

Figure 2 (Continued)

expressed by this antibody belongs to the $\mathrm{V}_{\mathrm{H}}$ III gene family (Table I). At the level of expression, RF-SJ3 shares the greatest homology $(95.8 \%)$ to the light chain $V_{L}$ gene segment of the BOR rheumatoid factor (data not shown). At the nucleotide level, the germline variable region gene HumKv325 is the most similar $(98.7 \%)$ to the $V_{L}$ region gene of RF-SJ3 (Fig. 2 and Table I) suggesting that HumKv325 or a closely related gene may have been used in this antibody. A distinct feature of this antibody is the presence of two additional amino acid residues (glycine) at positions 96 and 97 within CDR3. Studies are underway to address the possible mechanisms responsible for the generation of the extra proline residues found in CDR3 of antibodies RF-TS2 and RF-TMC1 and the extra glycine residues found in CDR3 of antibody RF-SJ3 and to determine whether these amino acids have a functional role in generating rheumatoid factor activity.

Two monospecific monoclonal rheumatoid factors use $V_{\lambda} I$ gene segments. RF-SJ 1 and RF-SJ 2 represent the first reported nucleotide sequences of $V_{\lambda} I$ rheumatoid factors (Fig. 5). Both of these rheumatoid factors were isolated from a patient with rheumatoid arthritis and express $V_{\lambda} I$ and $J_{\lambda} 2$ gene segments in association with a $\mathrm{V}_{\mathbf{H}}$ III heavy chain gene (Table I). At the level of light chain expression, these antibodies are most homologous to the FOG-B antibody (37) which is an antibody with
anti-D reactivity. RF-SJ 1 shares $88.0 \%$ homology at the amino acid level with FOG-B and RF-SJ2 shares $92.0 \%$ homology at the amino acid level with this antibody (Fig. 5 and Table I). These rheumatoid factors were compared to an expressed lambda light chain antibody as few germline variable lambda gene segments have been sequenced. Oligonucleotide probes specific for the CDR 1 regions of RF-SJ 1 and RF-SJ 2 were used to screen specific variable region gene subsets amplified from genomic DNA from the donor as well as from several normal individuals $(n=20)$. By Southern analysis, neither CDR 1 specific probe detected an appropriate variable region fragment in the donor or in a large number of normal individuals, clearly indicating that these antibodies have undergone somatic mutation.

\section{Discussion}

This study presents the first characterization of $V_{L}$ gene usage at the nucleotide level of monospecific, monoclonal IgM rheumatoid factors isolated from patients with autoimmune disorders. Most of the rheumatoid factors were isolated from patients with rheumatoid arthritis, and were generated by EBV transformation of B-lymphocytes obtained from inflamed sy-

CDR1

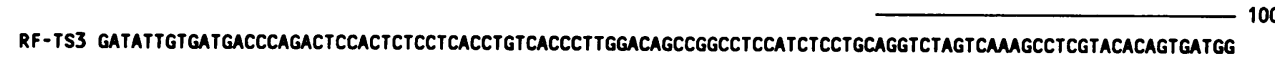

CDR2

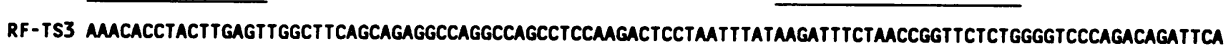

CDR3

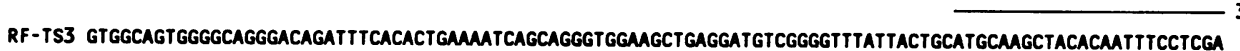

RF-TS3 $\overline{M C}$

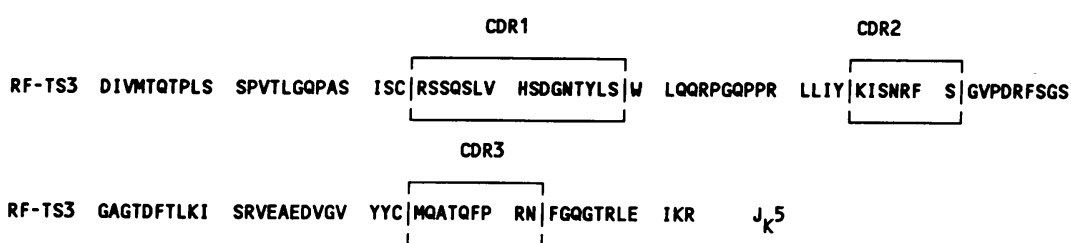

Figure 3. $(A)$ Nucleotide sequence of the variable gene segment of the $V_{K}$ II rheumatoid factor RF-TS3. The CDR regions are shown. $(B)$ Translated amino acid sequence of RF-TS3. The CDR regions are indicated and the $\mathrm{J}$ segment is shown. 


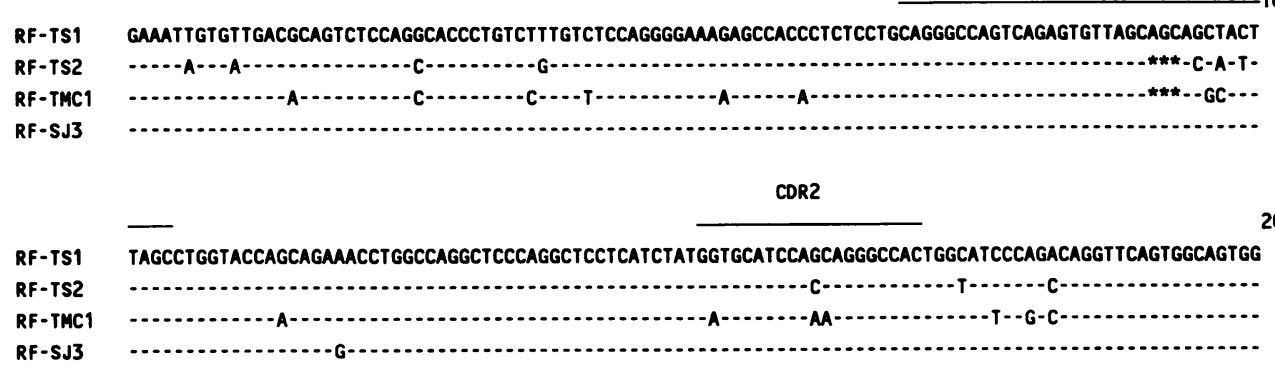

RF-SJ3

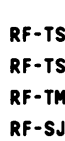

CDR3

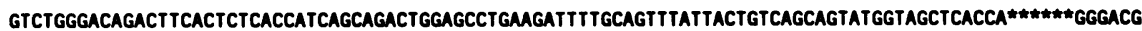

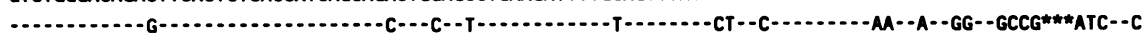

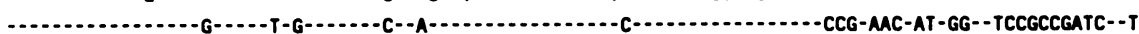
$-\mathbf{G}-$
B

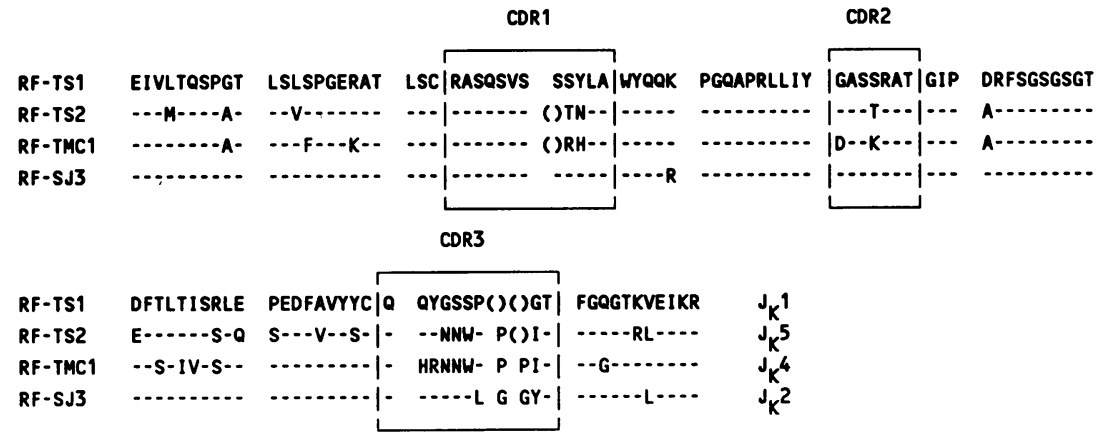

Figure 4. (A) Comparison of the nucleotide sequences of the $V_{L}$ gene segments of the $V_{K}$ III rheumatoid factors RF-TS1, RF-TS2, RFTMC1, and RF-SJ3. Areas of identical sequence are shown with dashes. Deletions are depicted by asterisks (*). The CDR regions are indicated above the sequence. $(B)$ Comparison of the deduced amino acid sequences of RF-TS1, RFTS2, RF-TMC1, and RF-

SJ3. Identical amino acids are indicated by dashes. Deletions within the CDR regions are shown by parenthesis. The particular $\mathbf{J}$ segment used is given. novial tissue. These rheumatoid factors are probably representative of the rheumatoid factors involved in the disease process as they are present at the site of active inflammation $(38,39)$. Unlike many previous studies of mixed cryoglobulin rheumatoid factors, the repertoire of $V_{L}$ gene segments used by these rheumatoid factors does not appear to be restricted providing additional evidence that the Wa and Po CRIs represent minor populations of rheumatoid factors in patients with autoimmune diseases. While one of the rheumatoid factors isolated from synovial tissue is an unmutated copy of a germline gene, most appear to have undergone some degree of somatic mutation.

Previous studies of mixed cryoglobulin rheumatoid factors showed that light chain variable region gene usage was re-
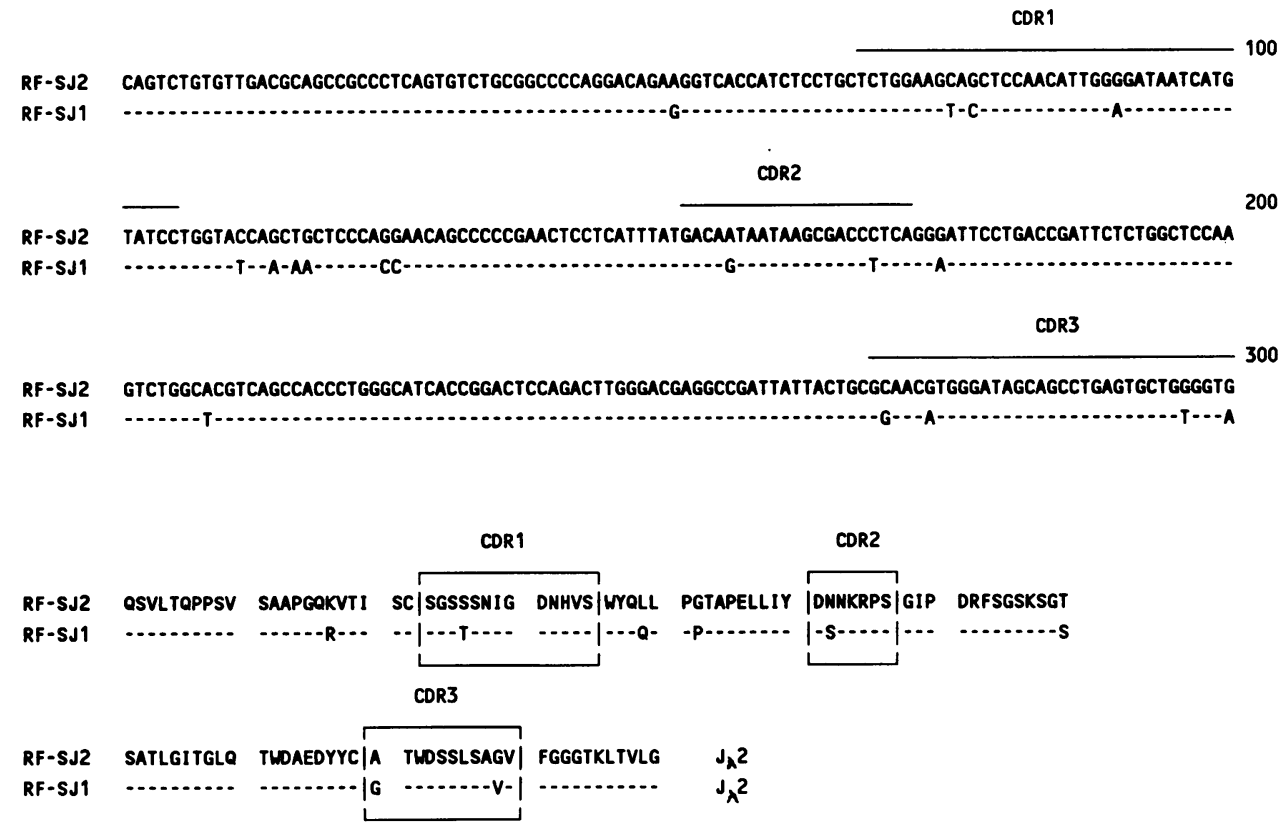

Figure 5. (A) Nucleotide sequence comparison of the $V_{L}$ gene segments of the $V_{\lambda} I$ rheumatoid factors RF-SJ1 and RF-SJ2. Dashes indicate identical sequence. The CDR regions are shown. $(B)$ Amino acid sequence comparison of the rheumatoid factors RF-SJ1 and RF-SJ2. Dashes indicate identical sequence and the $J$ segment used is shown. 
stricted, with a predominance of the Wa and Po CRI that define $\mathrm{V}_{\mathbf{K}}$ IIIb and $\mathrm{V}_{\mathbf{K}}$ IIIa specific variable region genes, respectively $(6,8,10,17,21)$. The variable region genes used in rheumatoid factors that express the Wa or Po CRIs are either identical to the germline sequences HumKv325 or HumKv328, respectively, or deviate from the germline sequence by only a few amino acid substitutions which may be due to genetic polymorphism and/or somatic mutation. However, in this study, the variable region genes of the rheumatoid factors isolated from patients with rheumatoid arthritis were representative of each of the $V_{K}$ families as well as the $V_{\lambda} I$ gene family. In this study, 2/9 rheumatoid factors use a $V_{K} I$ gene segment, $1 / 9$ express a $V_{K}$ II gene segment, 4/9 use $V_{K}$ III gene segments, and $2 / 9$ express $V_{\lambda} I$ gene segments. One $V_{\lambda}$ III rheumatoid factor has been described previously (40) providing additional evidence for the polyclonal nature of these rheumatoid factors. These data confirm a number of serological studies that demonstrated that the sera of patients with rheumatoid arthritis (26) contain little if any of the Wa or Po CRI, suggesting that either different variable region genes are being used in rheumatoid arthritis or that extensive somatic mutation has occurred in these antibodies $(16,21,25,26)$.

One of these rheumatoid factors expresses a $V_{L}$ gene segment that is a direct copy of a germline gene. The $V_{L}$ gene segment of antibody RF-TS 3 is derived from the germline gene A23 without mutation as its sequence at the nucleotide and amino acid level is identical to a previously described gene. RF-TS1 and RF-SJ3 probably arose from the germline gene HumKv325; the two nucleotide differences between RF-TS1 and HumKv325 may represent genetic polymorphism while the differences between RF-SJ3 and HumKv325 may arise from somatic mutation. While the origins of RF-KLl and RFTS2 are more problematic, they likely arise from the germline genes Vd and HumKv328, respectively, with a limited degree of somatic mutation. Collectively, these data imply that at least some potentially pathogenic autoantibodies are germline encoded or derive from germline genes with only limited amounts of somatic mutation. The rheumatoid factors RFKES1 and RF-TMC1 express light chain genes that do not exhibit a high degree of similarity to any known $V_{K}$ germline genes. These antibodies may use germline genes that are unique to the patient but more likely represent examples of somatic diversification. The rheumatoid factors RF-SJ1 and RF-SJ2 provide convincing evidence that somatic mutation occurs in true rheumatoid factors. Thus, RF-TS3 is a direct copy of a germline gene, while RF-KL1, RF-TS1, RF-TS2, and RF-SJ3 are derived from germline genes with only limited somatic mutation. The rheumatoid factors RF-KES1, RFTMC1, RF-SJ1, and RF-SJ2 are examples of antibodies where extensive somatic mutation has occurred. Collectively, these results argue that while some rheumatoid factors present in autoimmune disorders are direct copies of germline genes, most undergo somatic diversification.

Structural studies of mixed cryoglobulin rheumatoid factors revealed that variable region gene usage in both the heavy and light chains appeared to be restricted. The analysis of the heavy chains of the same monospecific rheumatoid factors presented in this study has been done in a parallel study from our laboratory (41). Pascual et al. have demonstrated that the $V_{H}$ gene families used by these rheumatoid factors are polyclonal in origin, similar to what is seen in the light chain (41). Not only do the $V_{H}$ and $V_{L}$ gene segments derive from a diverse population of germline genes, the $V_{H}$ gene segments are not those classically associated with the Wa or Po CRI. Several rheumatoid factors share the greatest homology with $\mathrm{V}_{\mathrm{H}}$ germline genes present in the fetal repertoire. The existence of somatic mutation is evident in some of the $V_{H}$ heavy chain genes supporting the role for somatic mutation in the generation of rheumatoid factor activity in patients with rheumatoid arthritis.

Rheumatoid factors RF-TS2, RF-TMC1, and RF-SJ3 contain additional amino acid (proline/glycine) residues within CDR3. There are very few examples in the literature in either the mouse or human antibody systems where variations in the length of CDR3 have been demonstrated. In the mouse, an antiphosphocholine antibody contains an extra proline residue (42), an antioxazalone antibody contains two extra amino acids, proline and methionine (43), and in a number of aberrant rearrangements additional amino acid residues have been seen $(44,45)$. In the human system, many examples can be found in lymphomas involving translocations within either chromosome(s) 2 or 22, the locations of the kappa and lambda light chain loci, respectively $(45,47)$. To date, there are only a few human antibodies that contain additional residues within CDR3. The antibody MEV, of unknown specificity, contains an extra glutamic acid residue (48). The most striking example is a mixed cryoglobulin SON (49) that expresses rheumatoid factor activity and in addition has LDL receptor reactivity. This antibody uses a $V_{K}$ IIIb variable region gene and contains an additional proline residue within CDR3 at amino acid position 96 . The light chain of SON has $97.0 \%$ homology at the amino acid level to RF-TS2 (data not shown).

As evidence for variation in the length of CDR3 is limited, studies are underway to examine possible mechanisms that could account for the generation of these residues. One explanation is that the extra residues are germline encoded at either the $3^{\prime}$ end of the variable region gene or the $5^{\prime}$ end of the joining segment. A second model would involve errors in heptamer/ nonamer recombination. Alternatively, these proline residues could be the result of $\mathrm{N}$-segment addition. While the codons for proline are GC-rich, characteristic of terminal deoxy transferase ( $\mathrm{TdT}$ ) activity, it is generally believed that TdT activity is not present at the time of light chain rearrangement. An attractive possibility is that there is an active mechanism responsible for generating these amino acid residues, and that these amino acids are necessary for rheumatoid factor activity. There are several examples where the somatic generation of a particular amino acid residue is correlated with antigen binding. For example, in the response to arsonate of the A/J mouse, intracodonic recombination generates an arginine residue at position 96 of the light chain that is required for arsonate binding (50). The functional importance of these proline residues in rheumatoid factor activity in this system is presently being analyzed.

While nine monoclonal IgM rheumatoid factors isolated from four patients and one normal individual were characterized in this study, obviously, it will be necessary to examine other rheumatoid factors isolated from additional patients with autoimmune disorders in order to address the issue of $V_{L}$ gene usage more completely. Since germline counterparts for most of the rheumatoid factors characterized in this study were not easily identified, Southern analysis using CDR or FW-specific oligonucleotides was done to address the question of whether the variable region gene being used was germline encoded or had undergone somatic mutation. While this type of analysis is not as definitive as isolating and sequencing the germline coun- 
terparts, in a multigenic system finding the precise germline counterpart can be a daunting task.

This study presents the structural analysis of a series of monospecific rheumatoid factors isolated from patients with several autoimmune disorders such as rheumatoid arthritis, polyarticular juvenile rheumatoid arthritis, and systemic lupus erythematosus. The light chain variable region gene segments used by these rheumatoid factors is not as restricted as previous work on mixed cryoglobulin rheumatoid factors suggested. Thus, these data argue that unlike the situation with mixed cryoglobulins, the Wa and Po CRIs represent only a minor population of the rheumatoid factors in patients with rheumatoid arthritis. While some of these rheumatoid factors use unmutated germline variable region genes, suggesting that at least some germline genes are capable of directly encoding potentially pathogenic antibodies, the vast majority had some degree of somatic diversification, arguing that processes such as antigenic selection and affinity maturation play a role in the immune response in autoimmune disease.

\section{Acknowledgments}

The authors are grateful to Virginia Pascual for helpful discussions.

Supported by grants from the National Institutes of Health (CA44016 and AR-39169) to J. D. Capra and AR-39254 and AR-34211 to S. M. Fu. I. Randen, O. Forre, and J. B. Natvig are supported by The Norwegian Women's Health Organization, The Norma and Leon Hess and The Grete Harbitz Legacies. J. D. Capra holds the Edwin L. Cox Distinguished Chair in Immunology and Genetics at the University of Texas Southwestern Medical Center.

\section{References}

1. Williams, R. C., Jr., and H. G. Kunkel. 1962. Rheumatoid factor, complement, and conglutinin aberrations in patients with subacute bacterial endocarditis. J. Clin. Invest. 41:666-675.

2. Koopman, J., and R. A. Schrohenloher. 1980. In vitro synthesis of IgM rheumatoid factor by lymphocytes from healthy donors. J. Immunol. 125:934 938.

3. Welch, M. J., S. Fong, J. H. Vaughan, and D. A. Carson. 1983. Increased frequency of rheumatoid factor-precursor B lymphocytes after immunization of normal adults with tetanus toxoid. Clin. Exp. Immunol. 51:299-304.

4. Panush, R. S., N. E. Bianco, and P. H. Schur. 1971. Serum and synovial fluid IgG, IgA and IgM antigammaglobulins in rheumatoid arthritis. Arthritis Rheum. 14:737-747.

5. Withrington, R. H., I. Teitsson, H. Valdimarsson, and M. H. Seifert. 1984 Prospective study of early rheumatoid arthritis. II. Association of rheumatoid factor isotypes with fluctuations in disease activity. Ann. Rheum. Dis. 43:679 685.

6. Kunkel, H. G., V. Agnello, F. G. Joslin, R. J. Winchester, and J. D. Capra 1973. Cross-idiotypic specificity among monoclonal IgM proteins with antigamma globulin activity. J. Exp. Med. 137:331-342.

7. Kunkel, H. G., R. J. Winchester, F. G. Joslin, and J. D. Capra. 1974. Similarities in the light chains of anti-gamma-globulins showing cross-idiotypic specificities. J. Exp. Med. 139:128-136.

8. Agnello, V., A. Arbetter, G. I. de Kasep, R. Powell, E. M. Tan, and F. Joslin. 1980. Evidence for a subset of rheumatoid factors that cross-react with DNA-histone and have a distinct cross-reactive idiotype. J. Exp. Med. 151:1514-1527.

9. Radoux, V., P. P. Chen, J. A. Sorge, and D. A. Carson. 1986. A conserved human germline $\mathrm{V}_{k}$ gene directly encodes rheumatoid factor light chains. J. Exp. Med. 164:2119-2124.

10. Chen, P. P., D. L. Robbins, F. R. Jirik, T. J. Kipps, and D. A. Carson. 1987. Isolation and characterization of a light chain variable region gene for human rheumatoid factors. J. Exp. Med. 166:1900-1905.

11. Chen, P. P., K. Albrandt, T. J. Kipps, V. Radoux, F. T. Liu, and D. A. Carson. 1987. Isolation and characterization of human kIII germ line genes: implications for the molecular basis of human kIII light chain diversity. J. Immunol 139:1727-1733.

12. Schrohenloher, R. E., and W. J. Koopman. 1986. An idiotype common to rheumatoid factors from patients with rheumatoid arthritis identified by a monoclonal antibody. Arthritis Rheum. 29:28S.

13. Crowley, J. J., R. D. Goldfien, R. E. Schrohenloher, H. L. Spiegelberg,
G. J. Silverman, R. A. Mageed, R. Jefferis, W. J. Koopman, D. A. Carson, and S. Fong. 1988. Incidence of three cross-reactive idiotypes on human rheumatoid factors paraproteins. J. Immunol. 140:3411-3418.

14. Capra, J. D., and J. M. Kehoe. 1974. Structure of antibodies with shared idiotypy: the complete sequence of the heavy chain variable regions of two immunoglobulin M anti-gamma globulins. Proc. Natl. Acad. Sci. USA. 71:4032-4036.

15. Silverman, G. J., F. Goñi, J. Fernandez, P. P. Chen, B. Frangione, and D. A. Carson. 1988. Distinct patterns of heavy chain variable region subgroup use by human monoclonal autoantibodies of different specificity. J. Exp. Med. 168:2361-2366.

16. Mageed, R. A., M. Dearlove, D. M. Goodall, and R. Jefferis. 1986. Immunogenic and antigenic epitopes of immunoglobulins. XVII. Monoclonal antiidiotypes to the heavy chain of human rheumatoid factors. Rheumatol. Int. 6:179-186.

17. Chen, P. P., K. Albrandt, N. K. Orida, V. Radoux, E. Y. Chen, R. Schrantz, F. Liu, and D. A. Carson. 1986. Genetic basis for the cross-reactive idiotypes of the light chains of human IgM anti-IgG autoantibodies. Proc. Natl. Acad. Sci. USA. 83:8318-8322.

18. Goldfien, R. D., P. Chen, T. J. Kipps, G. Starkebaum, J. G. Heitzmann, V. Radoux, S. Fong, and D. A. Carson. 1987. Genetic analysis of human B cell hybridomas expressing a cross-reactive idiotype. J. Immunol. 138:940-944.

19. Liu, M., D. L. Robbins, J. J. Crowley, S. Sinha, F. Kozin, T. J. Kipps, D. A. Carson, and P. P. Chen. 1989. Characterization of four homologous L chain variable region genes that are related to 6B6.6 idiotype positive human rheumatoid factor L chains. J. Immunol. 142:688-694.

20. Silverman, G. J., R. E. Schrohencoher, M. A. Accavihi, W. J. Koopman, and D. A. Carson. 1990. Structural characterization of the second major crossreactive idiotypic group of human rheumatoid factors: association with the $V_{H^{4}} 4$ gene family. Arthritis Rheum. 33:1-14.

21. Forre, O., J. H. Dobloug, T. E. Michaelsen, and J. B. Natvig. 1979. Evidence of similar idiotypic determinants on different rheumatoid factor populations. Scand. J. Immunol. 9:281-285.

22. Bonagura, V. R., L. Mendez, N. Agostino, and B. Pernis. 1987. Monomeric (7S) IgM found in the serum of rheumatoid arthritis patients share idiotypes with pentameric (19S) monoclonal rheumatoid factors. J. Clin. Invest. 79:813-818.

23. Bonagura, V. R., H. G. Kunkel, and B. Pernis. 1982. Cellular localization of rheumatoid factor idiotypes. J. Clin. Invest. 69:1356-1365.

24. D. N. Posnett. 1989. Idiotypes of rheumatoid factors. Monogr. Allergy. 26:111-119.

25. Mageed, R. A., and R. Jefferis. 1988. Analysis of rheumatoid factor autoantibodies in patients with essential mixed cryoglobulinemia and rheumatoid arthritis. Scand. J. Rheumatol. Suppl. 75:172-178.

26. Nelson, J. L., F. A. Nardella, I. R. Oppliger, and M. Mannik. 1987. Rheumatoid factors from patients with rheumatoid arthritis possess private repertoires of idiotypes. J. Immunol. 138:1391-1395.

27. Gaskin, F., B. S. Kingsley, and S. M. Fu. 1987. Autoantibodies to neurofibrillary tangles and brain tissue in Alzheimer's disease. Establishment of EpsteinBarr virus-transformed antibody-producing cell lines. J. Exp. Med. 165:245250.

28. Randen, I., K. M. Thompson, J. B. Natvig, O. Forre, and K. Waalen. 1989. Human monoclonal rheumatoid factors from the polyclonal repertoire of rheumatoid synovial tissue: production and characterization. Clin. Exp. Immunol. 78:13-18.

29. Thompson, K., M. Randen, J. B. Natvig, R. A. Mageed, R. Jefferis, D. A. Carson, H. Tighe, and O. Forre. 1990. Human monoclonal rheumatoid factors derived from the polyclonal repertoire of rheumatoid synovial tissue: incidence of cross-reactive idiotypes and expression of $\mathrm{V}_{\mathrm{H}}$ and $\mathrm{V}_{\mathrm{K}}$ subgroups. Eur. J. Immunol. 20:863-868.

30. I. M. Verma. 1981. The Enzymes: Nucleic Acids. Part A. XIV. P. D. Boyer, editor. Academic Press, New York. pp. 87-103.

31. Gubler, U., and B. J. Hoffman. 1983. A simple and very efficient method for generating cDNA libraries. Gene (Amst.). 25:263-294.

32. Saiki, R. K., D. H. Gelfand, S. Stoffel, S. J. Scharf, R. Higushi, G. T. Horn, K. B. Mullis, and H. A. Erlich. 1988. Primer-directed enzymatic amplification of DNA with a thermostable DNA polymerase. Science (Wash. DC). 239:487-491.

33. Okayama, H., and P. Berg. 1982. High-efficiency cloning of full-length cDNA. Mol. Cell. Biol. 2:161-170.

34. Sanger, F., A. R. Carlson, B. G. Barrel, A. J. H. Smith, and B. Roe. 1980. Cloning in single-stranded bacteriophage as an aid to rapid DNA sequencing. $J$. Mol. Biol. 143:161-178.

35. Scharf, S. J., G. T. Horn, and H. A. Erlich. 1986. Direct cloning and sequence analysis of enzymatically amplified genomic sequences. Science (Wash. DC). 233:1076-1078.

36. Maniatis, T., E. F. Fritsch, and J. Sambrook. 1982. Molecular Cloning: A Laboratory Manual. Cold Spring Harbor Laboratories, Cold Spring Harbor, New York.

37. Hughes-Jones, N. C., J. M. Bye, D. Beale, and J. Coadwell. 1990. Nucleotide sequences and three-dimensional modelling of the VH and VL domains of 
two human monoclonal antibodies specific for the $D$ antigen of the human $\mathbf{R h}$ blood group system. Biochem. J. 268:135-140.

38. Natvig, J. B., and E. Munthe. 1975. Self associating IgG rheumatoid factors represents a major response of plasma cells in rheumatoid inflammatory tissue. Ann. NY Acad. Sci. 256:88-95.

39. Munthe, E., and J. B. Natvig. 1972. Complement-fixing intracellular complexes of IgG rheumatoid factor in rheumatoid plasma cells. Scand. J. Immunol. 1:161-170.

40. Natvig J. B., K. Thompson, I. Randen, M. Steinitz, M. Taussig, D. Beale, P. Barker, K. Sletten, K. Waalen, and O. Forre. 1988. Partial amino acid sequence and variable subgroup determination (VH and VL) of monoclonal rheumatoid factor derived from a rheumatoid arthritis patient. Scand. J. Rheumatol. Suppl. 75:127-132.

41. Pascual, V., I. Randen, K. Thompson, M. Sould, O. Forre, J. Natvig, and J. D. Capra. 1990. The complete nucleotide sequences of the heavy chain variable regions of six monospecific rheumatoid factors derived from Epstein-Barr virustransformed B cells isolated from the synovial tissue of patients with rheumatoid arthritis. Further evidence that some autoantibodies are unmutated copies of germline genes. J. Clin. Invest. 86:1320-1328.

42. Weigert, M., L. Gatmaitan, E. Loh, J. Schilling, and L. Hood. 1978. Rearrangement of genetic information may produce immunoglobulin diversity. Nature (Lond.). 276:785-790.

43. Kaartinen, M., G. M. Griffiths, P. H. Hamlyn, A. F. Markham, K. Karjalainen, J. L. T. Pelkonen, O. Makela, and C. Milstein. 1983. Anti-oxazolone hybridomas and the structure of the oxazolone idiotype. J. Immunol. 130:937945.

44. Seidman, J. G., and P. Leder. 1980. A mutant immunoglobulin light chain is formed by aberrant DNA- and RNA-splicing events. Nature (Lond.). 286:779-783.

45. Roth, D. B., X. Chang, and J. H. Wilson. 1989. Comparison of filler DNA at immune, nonimmune, and oncogenic rearrangements suggests multiple mechanisms of formation. Mol. Cell. Biol. 9:3049-3057.

46. Heller, M., J. D. Owens, J. F. Mushinski, and S. Rudikoff. 1987. Amino acids at the site of $V_{k}-J_{k}$ recombination not encoded by germline sequences. $J$. Exp. Med. 166:637-646.

47. Finger, L. R., R. C. Harvey, R. C. A. Moore, L. C. Showe, and C. M. Croce. 1986. A common mechanism of chromosomal translocation in $\mathrm{T}$ - and B-cell neoplasia. Science (Wash. DC). 234:982-985.

48. Kabat, E. A., T. T. Wu, M. Reid-Miller, H. M. Perry, and K. S. Gottesman. 1987. Sequences of proteins of immunological interest. IV. Public Health Service, National Institutes of Health, Bethesda, MD.

49. Ledford, D. K., F. Goni, M. Pizzolato, E. C. Franklin, A. Solomon, and B. Frangione. 1983. Preferential association of kIIIb light chains with monoclonal human IgMk autoantibodies. J. Immunol. 131:1322-1325.

50. Sanz, I., and J. D. Capra. 1987. Vk and Jk gene segments of A/J Ars-A antibodies: somatic recombination generates the essential arginine at the junction of the variable and joining regions. Proc. Natl. Acad. Sci. USA. 84:1085-1089. 